09

\title{
О некоторых новых возможностях управления квантовыми системами с помощью униполярных предельно коротких импульсов
}

\author{
(C) P.М. Архипов ${ }^{1,2,3}$, М.В. Архипов ${ }^{1,2}$, А.В. Пахомов ${ }^{2}$, Н.Н. Розанов ${ }^{2,3,4}$ \\ ${ }^{1}$ Санкт-Петербургский государственный университет, \\ 199034 Санкт-Петербург, Россия \\ ${ }^{2}$ Университет ИТМО, \\ 197101 Санкт-Петербург, Россия \\ ${ }^{3}$ ФТИ им. А.Ф. Иофффе, \\ 194021 Санкт-Петербург, Россия \\ ${ }^{4}$ Государственный оптический институт им. С.И. Вавилова, \\ 199053 Санкт-Петербург, Россия \\ e-mail: arkhipovrostislav@gmail.com
}

Поступила в редакцию 12.09.2019 г.

В окончательной редакции 12.09.2019 г.

Принята к публикации 20.09.2019 г.

На основании приближенного решения уравнения Шредингера изучено воздействие пары предельно коротких, субцикловых импульсов малой амплитуды на квантовую систему. Показано, что в случае, когда длительность импульсов короче обратных частот атомных переходов, вероятность переходов между уровнями определяется электрической площадью импульса и временно́й задержкой между ними. Как следствие этого факта, униполярный импульс по сравнению с биполярным способен оказывать более эффективное воздействие на систему, а последовательность импульсов реализует селективное воздействие на квантовые объекты, несмотря на нерезонансный характер взаимодействия.

Ключевые слова: предельно короткие импульсы, униполярные импульсы, аттосекундные импульсы.

DOI: $10.21883 /$ OS.2020.01.48845.257-19

Прогресс в генерации предельно коротких импульсов (ПКИ) аттосекундной длительности сделал возможным изучать и управлять движением волновых пакетов в веществе на временах порядка периода колебаний световой волны [1-6]. При этом, если длительность импульса короче обратных частот резонансных переходов в системе, характер взаимодействия импульса со средой существенно зависит от формы импульса. В этом случае существенную роль играет „степень униполярности“ импульса, определяемая [7-9] как

$$
\xi=\frac{\left|\int E d t\right|}{\int|E| d t} .
$$

В числителе (1) стоит электрическая площадь импульca, которая определяется $[10,11]$ соотношением

$$
S_{E}=\int_{-\infty}^{+\infty} E(t) d t
$$

где $E$ - напряженность электрического поля и $t-$ время. Именно электрическая площадь импульса определяет характер взаимодействия ПКИ с микробъектами в случае, если длительность импульса короче периода резонансных переходов в атоме [7-9,12-17]. Действительно, униполярные ПКИ с ненулевой площадью позволяют оказывать однонаправленное воздействие на заряженные частицы, что открывает возможности для использования их для контроля динамики волновых пакетов в веществе $[4,7-9,12-15]$ и для ускорения заряженных частиц $[16,17]$. Методы получения униполярных импульсов описаны в обзорах [7-9] и цитируемой литературе. Квазиуниполярные импульсы полуцикловой формы получены экспериментально в оптическом [4-6] и в терагерцовом [18-21] диапазоне частот. Экспериментально изучалось взаимодействие субцикловых импульсов с веществом и возможность управление параметрами различных сред [4,22-25]. Так, в [4,25] показано, что результат действия полуцикловых, квазиуниполярных импульсов на среду отличается от случая действия биполярного многоциклового импульса.

Теоретически взаимодействие униполярных ПКИ с веществом практически не изучено на сегодняшний день. Данный вопрос требует тщательного теоретического анализа. Очевидно, что в случае, когда длительность импульса короче обратных частот атомных переходов, применение стандартного малоуровневого приближения может оказаться недостаточным для описания взаимодействия ПКИ с атомом. В этом случае теоретическое рассмотрение вопроса должно проводиться с помощью решения уравнения Шредингера для волновой функции квантовой системы [26]. В работах [12-15] на основе приближения внезапных возмущений (когда длительность импульса короче времени характерного движения электрона в атоме) изучалось действие ПКИ 
на простейшие квантовые объекты - атом водорода и гармонический осциллятор. В приближении внезапных возмущений считается, что за время действия импульса электрон не успевает заметно сместиться. Поэтому в первом приближении в уравнении Шредингера пренебрегается собственным гамильтонианом системы (оператором кинетической энергии и кулоновым полем ядра) во время действия поля импульса. В этом случае, как показывает анализ, вероятности переходов определяются электрической площадью импульса $[8,9,14,15]$. В случае, если длительность импульса сравнима или больше характерного времени движения электрона в атоме, для описания взаимодействия ПКИ с атомными системами применяют стандартную теорию возмущений $[26,27]$ или численное решение уравнение Шредингера [28,29] (см. также обзор [30] и цитируемую в нем литературу). Как показывает анализ [13], в случае малой длительности импульса результаты вычисления вероятности переходов, полученные с помощью приближения внезапных возмущений, приближенно совпадают с результатами расчетов, проведенными на основе стандартной теории возмущений в приближении слабого поля.

Целью данной заметки является анализ возможности управления состоянием атомной системы с помощью униполярных субцикловых ПКИ, длительность которых короче обратных частот атомных переходов в системе. Анализ проводится на основе стандартной теории возмущений, применимой для полей с амплитудой, меньшей внутриатомного поля $\left(\sim 10^{9} \mathrm{~V} / \mathrm{cm}\right)$. Будет показано, что вероятность перехода определяется электрической площадью импульса, а в случае воздействия на среду пары импульсов - задержкой между импульсами. А это, в свою очередь, показывает возможность более эффективного управления динамикой волновых пакетов с помощью униполярных ПКИ по сравнению с обычными биполярными импульсами.

Для теоретического описания воздействия ПКИ на квантовую систему необходимо использовать временное уравнение Шредингера для волновой функции $\psi[26]$ :

$$
i \hbar \frac{\partial \psi}{\partial t}=\left[\hat{H}_{0}+V(t)\right] \psi
$$

Здесь $\hbar-$ приведенная постоянная Планка, $\hat{H}-$ собственный гамильтониан системы и $V(t)=-d E(t)-$ потенциал ее взаимодействия с импульсом излучения в дипольном приближении, $d-$ дипольный момент атома. Предположим, что система взаимодействует с ПКИ малой амплитуды, меньшей внутриатомного поля, и длительность импульса короче обратных частот переходов в системе. В этом случае анализ взаимодействия будем проводить на основе приближенного решения уравнения Шредингера (3). Пусть до взаимодействия система была в основном состоянии $\Psi_{0}$ из дискретного набора собственных состояний гамильтониана $\hat{H}_{0}$. Вероятность перехода системы из основного состояния в возбужденные может быть вычислена [26] в первом порядке теории возмущений:

$$
w_{0 k}=\frac{1}{\hbar^{2}}\left|\int V_{0 k} e^{i \omega_{0 k} t}\right|^{2} .
$$

Здесь $V_{0 k}=-d_{0 k} E(t)$ - матричный элемент оператора возмущения, $d_{0 k}$ - дипольный момент перехода, $\omega_{0 k}-$ частота резонансного перехода.

Если длительность импульса короче обратной частоты атомного перехода, то осциллирующая экспонента под интегралом (4) не успеет заметно измениться за время действия импульса. Тогда при вычислении интеграла (4) в первом приближении можно положить в показателе экспоненты $t=0$. Данное приближение сводится к случаю, когда на систему действует импульс дельтаобразной формы. Тогда интеграл в (4) берется, и для вероятности перехода имеем

$$
w_{0 k}=\frac{d_{0 k}^{2}}{\hbar^{2}} S_{E}^{2}
$$

Из (5) видно, что вероятность перехода монотонно зависит от квадрата электрической площади импульса. Поэтому эффективность воздействия на систему униполярных ПКИ по сравнению с биполярными очевидна.

В случае действия на систему пары дельтаобразных импульсов, обладающих электрическими площадями $S_{E 1}$ и $S_{E 2}$, временной интервал между которыми $\Delta$, из формулы (4) нетрудно получить

$$
w_{0 k}=\frac{d_{0 k}^{2}}{\hbar^{2}}\left(S_{E 1}^{2}+S_{E 2}^{2}+2 S_{E 1} S_{E 2} \cos \omega_{0 k} \Delta\right) .
$$

Видно, что и в случае возбуждения системы парой импульсов результат воздействия определяется электрической площадью импульса и задержкой между импульсами. Если импульсы распространяются навстречу друг другу в протяженной среде с малой концентрацией частиц, то данная формула может быть интерпретирована как наведение гармонической решетки заселенностей, возникающей в протяженной среде [31-33]. Возможность наведения решеток в многоуровневой среде с помощью пары субцикловых импульсов изучалась авторами ранее в [33]. Однако если импульсы следуют друг за другом и воздействуют на тонкий слой частиц (или единичный атом), то данная формула иллюстрирует возможность управления населенностями между уровнями с помощью изменения задержки между импульсами. Таким образом, меняя задержку между импульсами, можно управлять состоянием среды, селективно возбуждать/девозбуждать те или иные переходы, несмотря на нерезонасный характер взаимодействия. Возможность эффективного, нерезонасного воздействия единичного униполярного импульса на квантовую систему (по сравнению с одноцикловым биполярным импульсом) была показана теоретически в [15]. А формула (6) по сути обобщает данный вывод на случай пары униполярных импульсов. 
Интересно отметить, что слагаемые в скобках по виду напоминают распределение освещенности при интерференции двух монохроматических волн в среде, где под $\Delta$ понимают разность хода двух волн [34]. Данное совпадение неудивительно, ведь результат возбуждения среды зависит от задержки между импульсами. Также зависимость вероятности от задержки между импульсами говорит о том, что результат воздействия каждого следующего импульса зависит от результата воздействия предыдущего импульса, т. е. от фазы волновой функции в момент прихода второго импульса, что характерно для когерентного взаимодействия импульса со средой (если длительность импульсов и задержка между ними короче времен релаксации в среде) [35].

Формулы (5) и (6) были получены в случае воздействия на систему дельтаобразных импульсов. Однако аналогичные выражения могут быть получены и в случае воздействия на систему импульсов произвольной формы. Пусть на систему воздействует пара униполярных импульсов гауссовой формы, имеющих в общем виде разные амплитуды и длительности (и соответственно площади):

$$
E(t)=E_{1} \exp \left[-t^{2} / \tau_{1}^{2}\right]+E_{2}\left[-(t-\Delta)^{2} / \tau_{2}^{2}\right] .
$$

Тогда вместо (6) имеем

$$
\begin{aligned}
w_{0 k} & =\frac{d_{0 k}^{2}}{\hbar^{2}} S_{E 1}^{2} \exp \left[-\frac{\omega_{0 k}^{2} \tau_{1}^{2}}{2}\right]+\frac{d_{0 k}^{2}}{\hbar^{2}} S_{E 2}^{2} \exp \left[-\frac{\omega_{0 k}^{2} \tau_{2}^{2}}{2}\right] \\
& +2 \frac{d_{0 k}^{2}}{\hbar^{2}} S_{E 1} S_{E 2} \exp \left[-\omega_{0 k}^{2}\left(\tau_{1}^{2}+\tau_{2}^{2}\right) / 4\right] \cos \left(\omega_{0 k} \Delta\right)
\end{aligned}
$$

$\left(S_{E 1}\right.$ и $S_{E 2}$ - площади импульсов). Из формулы (8) также видна периодическая зависимость заселенностей квантовых состояний системы от задержки между импульсами и ее зависимость от длительности импульсов. Таким образом, меняя задержку между импульсами и длительность импульсов, с помощью пары ПКИ можно оказывать селективное воздействие на квантовые объекты, несмотря на нерезонансный характер взаимодействия. Обратим внимание, что формула (8) применима к субцикловому импульсу с нулевой электрической площадью, состоящему из двух полуволн: первая полуволна обладает большой амплитудой и крайне малой длительностью, а вторая полуволна имеет большую длительность и малую амплитуду. Тогда в выражении $(8)$, если $\omega_{0 k} \tau_{2} \gg 1$, доминирующим будет являться первое слагаемое, а вторым и третьим слагаемым можно пренебречь. И действие такого одиночного субциклового импульса с нулевой площадью совпадает с действием одиночного униполярного импульса. Данный вывод качественно согласуется с экспериментальными результатами работы [4], в которой показано эффективное воздействие субциклового импульса на квантовую систему.

Приведем численные оценки. Пусть среда возбуждается аттосекундными импульсами гауссовой формы, длительность импульса $\tau_{p}=500 \mathrm{as,} \mathrm{амплитуда}$
$E_{0}=10^{7} \mathrm{~V} / \mathrm{cm}$, что сравнимо с экспериментальными результатами работы [4]. При $\omega_{0 k}=3 \cdot 10^{15} \mathrm{rad} / \mathrm{s}, d_{0 k}=15 \mathrm{D}$ имеем для максимального значения $w_{0 k} \sim 0.1$.

Таким образом, в работе на основании приближенного решения уравнения Шредингера проанализировано воздействие пары ПКИ на атомную систему в условиях, когда длительность импульса короче периодов резонансных переходов в атоме и его амплитуда слабее внутриатомного поля. Показано, что определяющую роль во взаимодействии играет электрическая площадь импульсов и временная задержка между ними. Это указывает на возможность селективного воздействия на среду с помощью измененения задержки между импульсами, несмотря не нерезонансный характер взаимодействия ПКИ со средой. Униполярные импульсы могут быть использованы для более эффективного управления динамикой волновых пакетов в веществе, химическими, а также ядерными реакциями, как отмечалось в работе [36], в которой сообщалось о первой экспериментальной регистрации униполярного электромагнитного излучения от пучка релятивистских электронов.

\section{Финансирование работы}

Изучение возможности сверхбыстрого управления состоянием среды с помощью пары импульсов выполнено при поддержке гранта РНФ 19-72-00012. Исследование роли электрической площади во взаимодействии выполнено при поддержке гранта РФФИ 19-02-00312а.

\section{Конфликт интересов}

Авторы заявляют, что у них нет конфликта интересов.

\section{Список литературы}

[1] Krausz F., Ivanov M. // Rev. Mod. Phys. 2009. V. 81. P. 163.

[2] Calegari F. etal. // J. Phys. B. 2016. V. 49. N 6. P. 062001.

[3] Ramasesha K., Leone S.R., Neumark D.M. // Ann. Rev. Phys. Chem. 2016. V. 67. P. 41.

[4] Hassan M.T., Luu T.T., Moulet A., Raskazovskaya O. etal. // Nature. 2016. V. 530. P. 66.

[5] Wu H.-C., Meyer-ter-Vehn J. // Nature Photon. 2012. V. 6. P. 304.

[6] Xu J., Shen B., Zhang X. et al. // Sci. Rep. 2018. V. 8. P. 2669.

[7] Архипов Р.М., Пахомов А.В., Архипов М.В., Бабушкин И., Толмачев Ю.А., Розанов Н.Н. // Письма в ЖЭТФ. 2017. T. 105. № 6. C. 388; Arkhipov R.M., Pakhomov A.V., Babushkin I., Tolmachev Yu.A., Rosanov N.N. // JETP Lett. 2017. V. 105. N 6. P. 408.

[8] Розанов Н.Н., Архипов М.В., Архипов Р.М., Веретенов Н.А., Пахомов А.В., Федоров С.В. // Опт. и спектр. 2019. T. 127. B. 1. C. 82; Veretenov N.A., Pakhomov A.V., Fedorov S.V. // Opt. Spectrosc. 2019. V. 127. N 1. P. 77.

[9] Архипов Р.М., Архипов М.В., Шимко А.А., Пахомов А.В., Розанов Н.Н. // Письма в ЖЭТФ. 2019. Т. 110. № 1. С. 9; Arkhipov R.M. Arkhipov M.V., Shimko A.A., Rosanov N.N. // JETP Lett. 2019. V. 110. N 1. P. 15. 
[10] Розанов Н.Н. // Опт. и спектр. 2009. Т. 107. № 5. С. 761; Rosanov N.N. // Opt. Spectrosc. 2009. V. 107. N 5. P. 721.

[11] Розанов Н.Н., Архипов Р.М., Архипов М.В. // УФН. 2018. T. 188. B. 12. C. 1347; Rosanov N.N., Arkhipov R.M. Arkhipov M.V. // Phys. Usp. 2018. V. 61. N 12. P. 1227.

[12] Dimitrovski D., Solov'ev E.A., Briggs J.S. // Phys. Rev. Lett. 2004. V. 93. P. 083003.

[13] Dimitrovski D., Solov'ev E.A., Briggs J.S. // Phys. Rev. A. 2005. V. 72. P. 043411.

[14] Розанов Н.Н. // Опт. и спектр. 2018. Т. 124. В. 1. С. 75; Rosanov N.N. // Opt. Spectrosc. 2018. V. 124. N 1. P. 72.

[15] Arkhipov R.M., Arkhipov M.V., Babushkin I.V., Demircan A., Morgner U., Rosanov N.N. // Opt. Lett. 2019. V. 44. N 5. P. 1202.

[16] Розанов Н.Н. // Опт. и спектр. 2018. Т. 125. В. 6. С. 818; Rosanov N.N. // Opt. Spectrosc. 2018. V. 125. N 6. P. 1012.

[17] Розанов Н.Н. // Опт. и спектр. 2019. Т. 126. В. 2. С. 211; Rosanov N.N. Opt. Spectrosc. 2019. V. 126. N 2. P. 140.

[18] Reiman K. // Rep. Progr. Phys. 2007. V. 70. P. 1597.

[19] Roskos H.G., Thomson M.D., Kress M., Loeffler T. // Laser Photon. Rev. 2007. V. 1. P. 349.

[20] Gao Y., Drake T., Chen Z., DeCamp M.F. // Opt. Lett. 2008. V. 33. P. 2776.

[21] Obraztsov P.A., Kaplas T., Garnov S.V., Kuwata-Gonokami M., Obraztsov A.N., Svirko Y.P. // Sci. Rep. 2014. V. 4. P. 4007.

[22] Jones R.R. // Phys. Rev. Lett. 1996. V. 76. P. 3927.

[23] Bensky T.J., Campbell M.B., Jones R.R. // Phys. Rev. Lett. 1998. V. 81. P. 3112.

[24] Wetzels A., Gjrtler A., Noordam L.D., Robicheaux F., Dinu C., Muller H.G., Vrakking M.J.J., van der Zande W.J. // Phys. Rev. Lett. 2002. V. 89. P. 273003.

[25] Chai X., Ropagnol X., Mohsen Raeis-Zadeh S., Reid M., Safavi-Naeini S., Ozaki T. // Phys. Rev. Lett. 2018. V. 121. P. 143901.

[26] Ландау Л.Д., Либиии Е.M. Квантовая механика. Нерелятивистская теория. М.: Наука, 1989. 768 с.; Landau L.D., Lifshitz E.M.. Quantum mechanics.Pergamon, 1974.

[27] Келдыш Л.В. // ЖЭТФ. 1964. Т. 47. С. 1945; Keldysh L.V. // Sov. Phys. JETP. 1965. V. 20. P. 1307.

[28] Kolesik M., Brown J.M., Moloney J.V., Faccio D. // Phys. Rev. A. 2014. V. 90. P. 033414.

[29] Karpel J.T., Yavuz D.D. // Opt. Lett. 2018. V. 43. P. 2583.

[30] Желтиков А.М. // УФН. 2017. Т. 187. В. 11. С. 1169-1204; Zheltikov A.M. // Phys. Usp. 2017. V. 60. N 11. P. 1087-1120.

[31] Arkhipov R.M., Arkhipov M.V., Babushkin I.V., Demircan A., Morgner U., Rosanov N.N. // Opt. Lett. 2016. V. 41. P. 4983.

[32] Arkhipov R.M., Pakhomov A.V., Arkhipov M.V, Babushkin I., Demircan A., Morgner U., Rosanov N.N. // Sci. Rep. 2017. V. 7. N 1. P. 12467.

[33] Архипов Р.М., Архипов М.В., Пахомов А.В., Розанов Н.Н. // Квант. электрон. 2019. Т. 49. № 10. C. 958-962; Arkhipov R.M., Arkhipov M.V., Pakhomov A.V., Rosanov N.N. // Quantum Electron. 2019. T. 49. N 10. P. $958-962$.

[34] Борн М., Вольф Э. Основы оптики. М.: Гл. ред. физ.-мат. лит-ры изд-ва „Наука“, 1973; Born M., Wolf E. Principles of optics: electromagnetic theory of propagation, interference and diffraction of light. Elsevier, 2013.

[35] Крюков П.Г., Летохов В.С. // УФН. 1969. Т. 99. В. 2. С. 169; Kryukov P.G., Letokhov V.S. // Sov. Phys. Usp. 1970. V. 12. P. H641.

[36] Naumenko G., Shevelev M. // J. Instrum. 2018. V. 13. C05001. 